\title{
Sharpening Bounds for Multilinear Schur Multipliers
}

\section{Anna Skripka ${ }^{1}$ (iD}

Received: 3 March 2021 / Revised: 7 November 2021 / Accepted: 8 November 2021 /

Published online: 19 January 2022

(c) The Author(s), under exclusive licence to Springer Science+Business Media LLC, part of Springer Nature 2022

\begin{abstract}
In this paper we make a brief survey of major estimates for norms and traces of multilinear Schur multipliers. We also discuss several open problems in operator theory and mathematical physics whose resolution could be reduced to sharpening existing bounds for Schur multipliers.
\end{abstract}

Keywords Multilinear Schur multiplier · Matrix function · Spectral shift · Positivity

Mathematics Subject Classification 47B49 $\cdot 15 \mathrm{~A} 45 \cdot 15 \mathrm{~A} 60 \cdot 15 \mathrm{~A} 69 \cdot 15 \mathrm{~A} 29$

\section{Introduction}

Schur multipliers and their generalizations have been studied for over a century. Linear Schur multipliers, which act on matrices as entrywise products, played a crucial role in Löwner's characterization of matrix monotone functions [1]. Further development of perturbation theory in [2] led to involvement of multilinear Schur multipliers, which are defined as intricate operations on tuples of matrices generalizing both the entrywise and usual products. Those transformations and their traces became fundamental objects in higher order perturbation theory. In particular, a recent resolution of several open questions in operator theory and mathematical physics relied on powerful upper bounds for (semi)norms of multilinear Schur multipliers (see, e.g., Chap. 5 in the book [3] or survey article [4]). In this paper we collect open questions closely related to establishing delicate lower bounds for multilinear Schur multipliers and relevant partial results that are scattered in the literature or have not been published.

This research was supported in part by NSF Grant DMS-1554456.

Anna Skripka

skripka@math.unm.edu

1 Department of Mathematics and Statistics, University of New Mexico, 311 Terrace Street North East, Albuquerque, NM 87106, USA 
In order to make our exposition accessible to a broader audience, we confine to finite-dimensional Schur multipliers. Although many of the questions stated below are motivated by infinite-dimensional problems, derivation of estimates for infinitedimensional multipliers often reduces to derivation of analogous estimates for simpler finite-dimensional multipliers. The interested reader can find a discussion of Schur multipliers on infinite-dimensional operators in the book [3], papers [5-7] and references cited therein.

Notations Assume that $d \in \mathbb{N}, d \geq 2$. Let $\mathcal{M}_{d}$ denote the space of all $d \times d$ matrices with entries in $\mathbb{C}$ and $\mathcal{M}_{d}(S)$ the set of $d \times d$ matrices with spectrum in $S \subset \mathbb{R}$. In particular, $\mathcal{M}_{d}(\mathbb{R})$ denotes the set of $d \times d$ self-adjoint (Hermitian) matrices and $\mathcal{M}_{d}[0, \infty)$ its subset of positive semidefinite matrices. Given $A, B, C \in \mathcal{M}_{d}(\mathbb{R})$, the notation $A \geq 0$ means that $A \in \mathcal{M}_{d}[0, \infty)$ and the notation $B \geq C$ means that $B-C \geq 0$. Let $\sigma(A)$ denote the spectrum, $\lambda_{\min }(A)$ the smallest eigenvalue and $\lambda_{\max }(A)$ the largest eigenvalue of $A \in \mathcal{M}_{d}(\mathbb{R})$.

Let $E_{i, j}$ denote the elementary matrix with the only nonzero entry in the position $(i, j)$, and $E_{j}$ the matrix $E_{j, j}$. We denote matrices by capital letters and their entries by the same low case letters, for instance, $A(i, j)=a_{i j}$ and $A_{k}=\left(a_{i j}^{(k)}\right)_{i, j=1}^{d}$ for $A, A_{k} \in \mathcal{M}_{d}$. Let $\operatorname{diag}\left(\alpha_{1}, \ldots, \alpha_{d}\right)$ denote the diagonal matrix whose $(i, i)$ th entry equals $\alpha_{i}$. Given $A=\left(a_{i j}\right)_{i, j=1}^{d} \in \mathcal{M}_{d}$, denote $\bar{A}=\left(\overline{a_{i j}}\right)_{i, j=1}^{d}, A^{T}=\left(a_{j i}\right)_{i, j=1}^{d}$, $A^{*}=\bar{A}^{T}$. The entrywise product of $A, B \in \mathcal{M}_{d}$ is denoted by $A \circ B$ and the usual product, when defined, by either $A B$ or $A \cdot B$.

The canonical inner product on $\mathbb{C}^{d}$ is denoted by $\langle\cdot, \cdot\rangle$ and the Euclidean norm by $\|\cdot\|_{2}$. The operator (spectral) norm of $A \in \mathcal{M}_{d}$ is denoted by $\|A\|$, the Schatten $p$-norm by $\|A\|_{p}:=\operatorname{Tr}\left(|A|^{p}\right)^{1 / p}, 1 \leq p<\infty$, and the vector $\ell^{\infty}$-norm by $\|A\|_{\ell_{\infty}\left(\mathbb{C}^{d^{2}}\right)}:=$ $\max _{1 \leq i, j \leq d}\left|a_{i j}\right|$. The space $\mathcal{M}_{d}$ equipped with the norm $\|\cdot\|_{p}$ is denoted $\mathcal{S}^{p}$. The norm of a multilinear transformation $T$ mapping the Cartesian product of Banach spaces $\mathcal{X}_{1}, \ldots \mathcal{X}_{n}$ to a Banach space $\mathcal{X}$ is denoted by

$$
\left\|T: \mathcal{X}_{1} \times \cdots \times \mathcal{X}_{n} \rightarrow \mathcal{X}\right\|
$$

We will work with functions $f$ in $C^{n}(S)$, the set of $n$-times continuously differentiable functions on an interval $S \subset \mathbb{R}$, and their divided differences. We recall that the divided difference of the zeroth order $f^{[0]}$ is the function $f$ itself. Let $\lambda_{0}, \lambda_{1}, \ldots, \lambda_{n}$ be points in $S$ and let $f \in C^{n}(S)$. The divided difference $f^{[n]}$ of order $n$ is defined recursively by

$$
f^{[n]}\left(\lambda_{0}, \ldots, \lambda_{n}\right)=\lim _{\lambda \rightarrow \lambda_{n}} \frac{f^{[n-1]}\left(\lambda_{0}, \ldots, \lambda_{n-2}, \lambda\right)-f^{[n-1]}\left(\lambda_{0}, \ldots, \lambda_{n-2}, \lambda_{n-1}\right)}{\lambda-\lambda_{n-1}} .
$$




\section{Bounds for Norms of Schur Multipliers and Taylor Remainders}

In this section we collect useful bounds for various norms and the trace-seminorm of rather general Schur multipliers and also discuss consequences of those bounds for Taylor remainders of matrix functions.

\subsection{Definition}

Let $n, d \in \mathbb{N}$ and let

$$
\mathfrak{m}(n):=\left\{m_{j_{0}, \ldots, j_{n}}\right\}_{j_{0}, \ldots, j_{n}=1}^{d}
$$

be a multidimensional matrix with complex entries. The $n$-linear Schur multiplier

$$
\mathfrak{M}_{\mathfrak{m}(n)}: \underbrace{\mathcal{M}_{d} \times \cdots \times \mathcal{M}_{d}}_{n \text { times }} \mapsto \mathcal{M}_{d}
$$

associated with the symbol $\mathfrak{m}(n)$ is defined in [8] by

$$
\mathfrak{M}_{\mathfrak{m}(n)}\left(X_{1}, \ldots, X_{n}\right):=\sum_{j_{0}, \ldots, j_{n}=1}^{d} m_{j_{0}, \ldots, j_{n}} \cdot x_{j_{0}, j_{1}}^{(1)} x_{j_{1}, j_{2}}^{(2)} \ldots x_{j_{n-1}, j_{n}}^{(n)} \cdot E_{j_{0}, j_{n}}
$$

where

$$
X_{k}=\left(x_{i, j}^{(k)}\right)_{i, j=1}^{d} \in \mathcal{M}_{d}, \quad k=1, \ldots, n
$$

The representation can be rewritten in the form

$$
\mathfrak{M}_{\mathfrak{m}(n)}\left(X_{1}, \ldots, X_{n}\right)=\sum_{j_{0}, \ldots, j_{n}=1}^{d} m_{j_{0}, \ldots, j_{n}} \cdot E_{j_{0}} X_{1} E_{j_{1}} X_{2} E_{j_{2}} \ldots X_{n} E_{j_{n}}
$$

When $n=1, \mathfrak{M}_{\mathfrak{m}(1)}$ is the classical Schur multiplier acting by $\mathfrak{M}_{\mathfrak{m}(1)}(X)=$ $\mathfrak{m}(1) \circ X$ and when $m_{j_{0}, \ldots, j_{n}}=1$ for all $j_{0}, \ldots, j_{n}=1, \ldots, d$, the transformation $\mathfrak{M}_{\mathfrak{m}(n)}$ acts as the usual matrix product $\mathfrak{M}_{\mathfrak{m}(n)}\left(X_{1}, \ldots, X_{n}\right)=X_{1} \ldots X_{n}$.

Let $n \in \mathbb{N}$ and $H_{0}, \ldots, H_{n} \in \mathcal{M}_{d}(\mathbb{R})$. Denote by $\left\{\lambda_{i}^{(k)}\right\}_{i=1}^{d}$ the complete list of eigenvalues of $H_{k}$ and by $\left\{\xi_{i}^{(k)}\right\}_{i=1}^{d}$ the respective orthonormal basis of eigenvectors of $H_{k}, k=0, \ldots, n$. Denote by $P_{\xi}$ the orthogonal projection on the unit vector $\xi$. Let $\varphi: \mathbb{R}^{n+1} \rightarrow \mathbb{C}$. Then, the multilinear Schur multiplier built over the spectral data of $H_{0}, \ldots, H_{n}$ is the transformation

$$
T_{\varphi}^{H_{0}, \ldots, H_{n}}: \mathcal{M}_{d} \times \cdots \times \mathcal{M}_{d} \rightarrow \mathcal{M}_{d}
$$


defined by

$$
\begin{aligned}
T_{\varphi}^{H_{0}, H_{1}, \ldots, H_{n}}\left(X_{1}, X_{2}, \ldots, X_{n}\right) \\
\quad:=\sum_{j_{0}, j_{1}, \ldots, j_{n}=1}^{d} \varphi\left(\lambda_{j_{0}}, \lambda_{j_{1}}, \ldots, \lambda_{j_{n}}\right) P_{\xi_{j_{0}}^{(0)}} X_{1} P_{\xi_{j_{1}}^{(1)}} X_{2} P_{\xi_{j_{2}}^{(2)}} \ldots X_{n} P_{\xi_{j_{n}}^{(n)}}
\end{aligned}
$$

for $X_{1}, \ldots, X_{n} \in \mathcal{M}_{d}$. The function $\varphi$ is called a symbol or integrand of the transformation $T_{\varphi}^{H_{0}, \ldots, H_{n}}$.

The representation (2.3) can be rewritten in terms of the spectral measures of $H_{0}, \ldots, H_{n}$. We recall that the spectral measure of a matrix $H \in \mathcal{M}_{d}(\mathbb{R})$ with the complete list of eigenvalues $\left\{\lambda_{i}\right\}_{i=1}^{d}$ and the respective orthonormal basis of eigenvectors $\left\{\xi_{i}\right\}_{i=1}^{d}$ is defined by $E_{H}(\delta):=\sum_{\lambda_{i} \in \delta} P_{\xi_{i}}$, where $\delta$ is a Borel subset of $\mathbb{R}$. If $\sigma\left(H_{k}\right)=\left\{\mu_{j}^{(k)}\right\}_{j=1}^{d_{k}}, d_{k} \leq d$, we obtain

$$
\begin{aligned}
& T_{\varphi}^{H_{0}, \ldots, H_{n}}\left(X_{1}, \ldots, X_{n}\right) \\
& =\sum_{j_{0}=1}^{d_{0}} \sum_{j_{1}=1}^{d_{1}} \ldots \sum_{j_{n}=1}^{d_{n}} \varphi\left(\mu_{j_{0}}^{(0)}, \mu_{j_{1}}^{(1)}, \ldots, \mu_{j_{n}}^{(n)}\right) E_{H_{0}}\left(\left\{\mu_{j_{0}}^{(0)}\right\}\right) X_{1} E_{H_{1}}\left(\left\{\mu_{j_{1}}^{(1)}\right\}\right) \ldots \\
& X_{n} E_{H_{n}}\left(\left\{\mu_{j_{n}}^{(n)}\right\}\right) .
\end{aligned}
$$

The transformation given by (2.3) can be interpreted as the transformation (2.1) (see Lemma 2.2), but derivation of results is still based on the former formula.

Notations 2.1 Let $n \in \mathbb{N}$, let $H_{0}, \ldots, H_{n} \in \mathcal{M}_{d}(\mathbb{R})$, and let $\left\{\lambda_{i}^{(k)}\right\}_{i=1}^{d}$ be the complete list of eigenvalues of $H_{k}$. Let $\varphi: \mathbb{R}^{n+1} \rightarrow \mathbb{C}$ and define

$$
\mathfrak{m}(n)=\left\{\varphi\left(\lambda_{j_{0}}^{(0)}, \ldots, \lambda_{j_{n}}^{(n)}\right)\right\}_{j_{0}, \ldots, j_{n}=1}^{d}
$$

Lemma 2.2 Assume Notations 2.1. Let $X_{1}, \ldots, X_{n} \in \mathcal{M}_{d}$. Let $\mathfrak{g}^{(k)}$ be an orthonormal basis of eigenvectors of $A_{k}$ with the corresponding d-tuple of eigenvalues $\left\{\lambda_{i}^{(k)}\right\}_{i=1}^{d}$, $k=0, \ldots, n$, and suppose that $X_{k}$ has the matrix representation $\left(x_{i j}^{(k)}\right)_{i, j=1}^{d}$ in the bases $\left\{\mathfrak{g}^{(k)}, \mathfrak{g}^{(k-1)}\right\}, k=1, \ldots, n$. The matrix representation of the transformation (2.4) in the bases $\left\{\mathfrak{g}^{(n)}, \mathfrak{g}^{(0)}\right\}$ coincides with the value of the Schur multiplier given by (2.1) on the $n$-tuple $\left(\left(x_{i j}^{(1)}\right)_{i, j=1}^{d}, \ldots,\left(x_{i j}^{(n)}\right)_{i, j=1}^{d}\right)$, that is,

$$
\mathfrak{M}_{\mathfrak{m}(n)}\left(X_{1}, \ldots, X_{n}\right)=T_{\varphi}^{H_{0}, \ldots, H_{n}}\left(X_{1}, \ldots, X_{n}\right)
$$

\subsection{Estimates for General Schur Multipliers}

The quality of the norm estimate for $T_{\varphi}^{H, \ldots, H}: \mathcal{X}_{1} \times \cdots \times \mathcal{X}_{n} \rightarrow \mathcal{X}$ given by (2.3) depends on the choice of the norms on the Banach spaces $\mathcal{X}_{1}, \ldots, \mathcal{X}_{n}, \mathcal{X}$. The best 
estimate (in fact, equality) holds for the Hilbert-Schmidt norm. Analogous estimates also hold for Schatten $p$-norms, $p>1$, with constant depending on $p$ while the dimension independent estimates for the operator and trace class norms of $T_{\varphi}^{H, \ldots, H}$ : $\mathcal{X}_{1} \times \cdots \times \mathcal{X}_{n} \rightarrow \mathcal{X}$ depend on a larger norm of $\varphi$. The latter case is not addressed in this paper; the interested reader can find the respective discussion in [3].

The proof of the following result can be found in [3, Proposition 4.1.3].

Theorem 2.3 Let $n \in \mathbb{N}$, let $H_{0}, \ldots, H_{n} \in \mathcal{M}_{d}(\mathbb{R})$, and let $\left\{\lambda_{i}^{(j)}\right\}_{i=1}^{d}$ be the complete list of eigenvalues of $H_{j}, j=0, \ldots, n$. Then, for a function $\varphi: \mathbb{R}^{n+1} \rightarrow \mathbb{C}$,

$$
\left\|T_{\varphi}^{H_{0}, \ldots, H_{n}}: \mathcal{S}^{2} \times \cdots \times \mathcal{S}^{2} \rightarrow \mathcal{S}^{2}\right\|=\max _{1 \leq j_{0}, \ldots, j_{n} \leq d}\left|\varphi\left(\lambda_{j_{0}}^{(0)}, \ldots, \lambda_{j_{n}}^{(n)}\right)\right|
$$

As a consequence of Theorem 2.3 we obtain the following upper bound for the seminorm $|\operatorname{Tr}(\cdot)|$ of the transformation (2.3).

Corollary 2.4 Let $n \in \mathbb{N}$, let $H, X_{1}, \ldots, X_{n} \in \mathcal{M}_{d}(\mathbb{R})$. Then, for a function $\varphi$ : $\mathbb{R}^{n+1} \rightarrow \mathbb{C}$,

$$
\left|\operatorname{Tr}\left(T_{\varphi}^{H, \ldots, H}\left(X_{1}, \ldots, X_{n}\right)\right)\right| \leq\|\varphi\|_{\infty}\left\|X_{1}\right\|_{2} \ldots\left\|X_{n}\right\|_{2}
$$

Proof By cyclicity of the trace,

$$
\operatorname{Tr}\left(T_{\varphi}^{H, \ldots, H}\left(X_{1}, \ldots, X_{n}\right)\right)=\operatorname{Tr}\left(T_{\psi}^{H, \ldots, H}\left(X_{1}, \ldots, X_{n-1}\right) X_{n}\right),
$$

where $\psi\left(\lambda_{0}, \ldots, \lambda_{n-1}\right)=\varphi\left(\lambda_{0}, \ldots, \lambda_{n-1}, \lambda_{0}\right)$. Applying Hölder's inequality in (2.7) yields

$$
\left|\operatorname{Tr}\left(T_{\varphi}^{H, \ldots, H}\left(X_{1}, \ldots, X_{n}\right)\right)\right| \leq\left\|T_{\psi}^{H, \ldots, H}\left(X_{1}, \ldots, X_{n-1}\right)\right\|_{2}\left\|X_{n}\right\|_{2} .
$$

Applying Theorem 2.3 on the right hand side of (2.8) yields the result.

Estimates for unitarily invariant norms of multilinear Schur multipliers have been extensively studied in the case when $\varphi$ equals the $n$th order divided difference $f^{[n]}$ of an $n$-times differentiable function $f$ or, more generally, equals a so-called polynomial integral momentum.

The following result is a particular case of [9, Theorem 5.6] for finite-dimensional operators and $\varphi=f^{[n]}$.

Theorem 2.5 Let $n \in \mathbb{N}$, let $H_{0}, \ldots, H_{n} \in \mathcal{M}_{d}(\mathbb{R})$, and let $1<\alpha, \alpha_{1}, \ldots, \alpha_{n}<\infty$ satisfy $\frac{1}{\alpha}=\frac{1}{\alpha_{1}}+\cdots+\frac{1}{\alpha_{n}}$. Then,

$$
\left\|T_{f^{[n]}}^{H_{0}, \ldots, H_{n}}: \mathcal{S}^{\alpha_{1}} \times \cdots \times \mathcal{S}^{\alpha_{n}} \rightarrow \mathcal{S}^{\alpha}\right\| \leq c_{\alpha_{1}, \ldots, \alpha_{n}}\left\|f^{(n)}\right\|_{\infty}
$$

for every $f \in C^{n}(\mathbb{R})$. 


\subsection{Estimates for Operator Taylor Remainders}

Let $H, V \in \mathcal{M}_{d}(\mathbb{R})$, let $(a, b)$ contain $\sigma(H) \cup \sigma(H+V)$. Then, for every $f \in$ $C^{n}[a, b]$, we define the $n$th Taylor remainder

$$
R_{n, f, H}(V):=f(H+V)-\left.\sum_{k=0}^{n-1} \frac{1}{k !} \frac{\mathrm{d}^{k}}{\mathrm{~d} t^{k}} f(H+t V)\right|_{t=0}
$$

The Fréchet derivatives of matrix functions in (2.10) are known to exist; see, for instance, [3, Sect. 5.3.1] for details.

Estimates for operator Taylor remainders are usually derived from the following representations in terms of multilinear Schur multipliers, which can be established by scrutinizing the proofs of [3, Theorems 5.3.2 and 5.4.3].

Theorem 2.6 Let $H, V \in \mathcal{M}_{d}(\mathbb{R})$, let $(a, b)$ contain $\sigma(H) \cup \sigma(H+V)$, and let $n \in \mathbb{N}$. Then, for every $f \in C^{n}[a, b]$,

$$
\left.\frac{\mathrm{d}^{n}}{\mathrm{~d} t^{n}} f(H+t V)\right|_{t=0}=T_{f^{[n]}}^{H, H, \ldots, H}(V, \ldots, V)
$$

and

$$
\begin{aligned}
R_{n, f, H}(V) & =T_{f^{[n]}}^{H+V, H, \ldots, H}(V, \ldots, V) \\
& =\frac{1}{(n-1) !} \int_{0}^{1}(1-t)^{n-1} T_{f^{[n]}}^{H+t V, H+t V, \ldots, H+t V}(V, \ldots, V) d t .
\end{aligned}
$$

As a consequence of Corollary 2.4 and the representation (2.12), we immediately obtain the following bound.

Proposition 2.7 Let $H, V \in \mathcal{M}_{d}(\mathbb{R})$, let $(a, b)$ contain $\sigma(H) \cup \sigma(H+V)$, and let $n \geq 2$ be an integer. Then the Taylor remainder $R_{n, f, H}(V)$ given by (2.10) satisfies the bounds

$$
\sup _{\substack{f \in C^{n}[a, b] \\\left\|f^{(n)}\right\|_{\infty} \leq 1}}\left|\operatorname{Tr}\left(R_{n, f, H}(V)\right)\right| \leq \frac{1}{n !}\|V\|_{2}^{n}
$$

The following estimate (and also its counterpart in the infinite-dimensional case) is derived in [9]. In [10, Theorem 4.1], that estimate is extended to $C^{n}(\mathbb{R})$. The respective details can be found in [3, Theorem 5.4.5].

Theorem 2.8 Let $n \in \mathbb{N}, 1<p<\infty$, and let $H, V \in \mathcal{M}_{d}(\mathbb{R})$. Then,

$$
\left\|R_{n, f, H}(V)\right\|_{p} \leq c_{n, p}\left\|f^{(n)}\right\|_{\infty}\|V\|_{p n}^{n}
$$

for every $f \in C^{n}(\mathbb{R})$. 
The inequality (2.14) does not hold for $p=1$. The result below is established in the course of the proof of [10, Lemma 5.10].

Theorem 2.9 Let $n \in \mathbb{N}$. Then, there exists $f_{n} \in C^{n}(\mathbb{R})$ such that for every $m \in \mathbb{N}$, there exist $H_{m}, V_{m} \in \mathcal{M}_{d_{m}}(\mathbb{R})$, where $d_{m}=4 m(n+1)^{2}$, such that $\sigma\left(H_{m}\right) \cup \sigma\left(H_{m}+\right.$ $\left.V_{m}\right)$ is contained in $\left[-1 / 2-e^{-1}, e^{-1}+1 / 2\right]$ and

$$
\left\|R_{n, f_{n}, H_{m}}\left(V_{m}\right)\right\|_{1} \geq c \sqrt{\log (4 m)}\left\|V_{m}\right\|_{n}^{n}
$$

\subsection{Spectral Shift Functions}

The result stated below was initially motivated by research in physics [11] and has a long history summarized in [3, Sect. 5.5]. In the infinite-dimensional setting, the result was established in $[9,12,13]$ for $n=1, n=2, n \geq 3$, respectively, while its finite-dimensional counterpart was derived by simpler methods. The result in the finite-dimensional setting follows from [14, Remark 3.2], [14, Theorem 4.3], [15, Theorem 5.1] for $n=1, n=2, n \geq 3$, respectively. While the class of functions in [15, Theorem 5.1] is more restrictive than in the result below, it can be extended by an approximation argument presented in the course of the proof of [16, Theorem 2.1].

Theorem 2.10 Let $H, V \in \mathcal{M}_{d_{m}}(\mathbb{R})$ and let $(a, b)$ contain $\sigma(H) \cup \sigma(H+V)$. Then, for every $n \in \mathbb{N}$, there exists unique $\eta_{n}=\eta_{n, H, V} \in L^{1}(R)$ supported in the convex hull of $\sigma(H) \cup \sigma(H+V)$ such that

$$
\operatorname{Tr}\left(R_{n, f, H}(V)\right)=\int_{\mathbb{R}} f^{(n)}(t) \eta_{n}(t) \mathrm{d} t
$$

for every $f \in C^{n}[a, b]$.

The spectral shift function $\eta_{n}$ satisfying Theorem 2.10 is a fundamental object in perturbation theory. In the finite-dimensional case, $\eta_{1}$ literally describes the shift of the spectrum of $H$ under the influence of a perturbation, while $\eta_{n}$ with $n \geq 2$ also encodes the rotation of eigenvalues. The following explicit formulas for $\eta_{n}$ are explained in [14] for $n=1,2$ and derived in [15] for $n \geq 3$.

Theorem 2.11 Let $H, V \in \mathcal{M}_{d}(\mathbb{R})$ and let $[a, b]$ be the convex hull of $\sigma(H) \cup \sigma(H+$ $V)$. Then, the following assertions hold.

(a)

$$
\eta_{1}(t)=\operatorname{Tr}\left(E_{H}((-\infty, t))\right)-\operatorname{Tr}\left(E_{H+V}((-\infty, t))\right)
$$

(b) For a.e. $t \in \mathbb{R}$,

$$
\eta_{2}(t)=\operatorname{Tr}\left(E_{H}((a, t)) V\right)-\int_{a}^{t} \eta_{1}(s) d s .
$$


(c) Let $n \geq 3$ be an integer. Then, for a.e. $t \in \mathbb{R}$,

$$
\begin{aligned}
\eta_{n}(t)= & \frac{\operatorname{Tr}\left(V^{n-1}\right)}{(n-1) !}-\int_{a}^{t} \eta_{n-1}(s) d s-\frac{1}{(n-1) !} \\
& \int_{\mathbb{R}^{n-1}}\left((x-t)_{+}^{n-2}\right)^{[n-2]}\left(x_{1}, \ldots, x_{n-1}\right) d \omega_{n-1}\left(\left(x_{1}, \ldots, x_{n-1}\right)\right),
\end{aligned}
$$

where $\omega_{k}$ is a finite Borel measure on $\mathbb{R}^{k}, k \in \mathbb{N}$, extending the set function

$$
\omega_{k}\left(A_{1} \times \cdots \times A_{k}\right):=\operatorname{Tr}\left(E_{H}\left(A_{1}\right) V \cdots E_{H}\left(A_{k}\right) V\right),
$$

where $A_{1}, \ldots, A_{k}$ are Borel subsets of $\mathbb{R}$. The measure $\omega_{k}$ is supported on $\sigma(H) \times$ $\cdots \times \sigma(H)$, so the second integral in (2.17) is, in fact, a finite sum.

\section{Inverse Perturbation Problem}

In this section we discuss lower bounds for Taylor remainders in terms of perturbations that provide a theoretical limitation on the accuracy of Taylor approximations of matrix functions. We also discuss reconstruction of an unknown perturbation from the spectral shift functions introduced in Sect. 2.4.

\subsection{Stability and Uniqueness}

The stability of a nonlinear inverse problem on recovering information about an unknown perturbation from information about the remainder was studied in [16]. One of the formulations of the respective problem is given below.

Question 3.1 Let $H, V \in \mathcal{M}_{d}(\mathbb{R})$, let $n \geq 2$, and let $(a, b) \supset \sigma(H) \cup \sigma(H+V)$. Find the largest constant $c_{n, d} \geq 0$ satisfying the bound

$$
\sup _{\substack{f \in C^{n}[a, b] \\\left\|f^{(n)}\right\|_{\infty} \leq 1}}\left|\operatorname{Tr}\left(R_{n, f, H}(V)\right)\right| \geq c_{n, d}\|V\|_{2}^{n} .
$$

Remark 3.2 (i) We note that the case $n=1$ is excluded from Question 3.1 since for every $H \in \mathcal{M}_{d}(\mathbb{R})$ there exist uncountably many $V \in \mathcal{M}_{d}(\mathbb{R})$ satisfying the property $\operatorname{Tr}(f(H+V))=\operatorname{Tr}(f(H))$ (see [16, Remark 2.8]). In other words, the left hand side of (3.1) equals 0 while the right hand side of (3.1) is positive for uncountably many $V$.

(ii) The search of the constant $c_{n, d}$ satisfying Question 3.1 might be simplified upon imposing suitable restrictions on $H, V, d$.

The following upper bound for $c_{n, d}$ satisfying (3.1) immediately follows from (2.13). 
Proposition 3.3 Under the assumptions of Question 3.1,

$$
c_{n, d} \leq(n !)^{-1} \text {. }
$$

It is established in [16] that if the left hand side of (3.1) is zero, then $V=0$. In fact, the following stronger result due to [16, Theorem 4.2] holds.

Theorem 3.4 Let $H, V \in \mathcal{M}_{d}(\mathbb{R})$. Then the following assertions are equivalent:

(i) $\operatorname{Tr}\left(R_{n, H, V}\left(t \mapsto t^{k}\right)\right)=0$ for every $k=n, \ldots, 2 d n-1$.

(ii) $\eta_{n}=0$ a.e.

(iii) $V=0$.

The result of Theorem 3.4 was applied in [16, Theorem 4.3] to characterize the equality of two graphs. We recall that two graphs are called isospectral if the spectra of their adjacency matrices coincide. A walk of length $k$ on a graph is a sequence of $k+1$ vertices each consecutive pair of which is connected by an edge. A self-returning walk is a walk whose starting and terminal vertices coincide.

Theorem 3.5 Let $G_{H}$ and $G_{K}$ be two graphs on a common set of $d$ vertices and with the adjacency matrices $H$ and $K$, respectively. Let $w_{H, k}$ denote the number of selfreturning walks of length $k$ on $G_{H}, w_{K, k}$ the number of self-returning walks of length $k$ on $G_{K}$, and $w_{K, 1, H, k-1}$ the number of self-returning walks of length $k$ where the first step is made along an edge of $G_{K}$ and the remaining $k-1$ steps are made along edges of $G_{H}$. Then, the graphs $G_{H}$ and $G_{K}$ are equal if and only if

$$
k w_{K, 1, H, k-1}=w_{K, k}+(k-1) w_{H, k}, \quad k=2, \ldots, 4 d-1 .
$$

If, in addition, $G_{H}$ and $G_{K}$ are isospectral, then they are equal if and only if

$$
w_{K, 1, H, k-1}=w_{H, k}, \quad k=2, \ldots, d+1 .
$$

The following lower bound follows from Theorem 2.9.

Proposition 3.6 Let $n \in \mathbb{N}$. Then, for every $m \in \mathbb{N}$, there exist $H_{m}, V_{m} \in \mathcal{M}_{d_{m}}(\mathbb{R})$, where $d_{m}=4 m(n+1)^{2}$, such that $\sigma\left(H_{m}\right) \cup \sigma\left(H_{m}+V_{m}\right) \subset\left[-1 / 2-e^{-1}, e^{-1}+1 / 2\right]$ and

$$
\sup _{\substack{f \in C^{n}[a, b] \\\left\|f^{(n)}\right\|_{\infty} \leq 1}}\left\|R_{n, f, H_{m}}\left(V_{m}\right)\right\|_{1} \geq c \sqrt{\log (4 m)} d_{m}^{1-\frac{n}{2}}\left\|V_{m}\right\|_{2}^{n} .
$$

If we compare the bound (3.4) to the one in Question 3.1, we note that the former holds for the larger norm $\|\cdot\|_{1}$ of the remainder and for selected $H, V \in \mathcal{M}_{d}(\mathbb{R})$. Passing to the smaller seminorm $|\operatorname{Tr}(\cdot)|$ and dropping the logarithmic factor allowed to obtain an analogous bound for all $H, V \in \mathcal{M}_{d}(\mathbb{R})$ in the case of an even $n$ in [16, Theorem 3.1], which is stated below. 
Theorem 3.7 Let $n$ be even. Then, under the assumptions of Question 3.1,

$$
c_{n, d} \geq(n !)^{-1} d^{1-\frac{n}{2}}
$$

Estimates analogous to (3.1) were also derived in the case of an odd $n$, but they depend on the sparsity of the set $\sigma(H)$. The following result is due to [16, Theorem 3.4].

Theorem 3.8 Let $H, V \in \mathcal{M}_{d}(\mathbb{R})$, let $n \geq 3$ be an odd integer. Let $\sigma(H)=\left\{\lambda_{1}<\right.$ $\left.\cdots<\lambda_{m}\right\}$, where $m \leq n$. If $\sigma(H)=\sigma(H+V)$, then

$$
\sup _{\substack{f \in C^{n}[a, b] \\\left\|f^{(n)}\right\|_{\infty} \leq 1}}\left|\operatorname{Tr}\left(R_{n, H, V}(f)\right)\right| \geq \frac{s_{H}\left(\lambda_{m}-\lambda_{1}\right)^{1-n} d^{1-n}}{2^{n+2}(n-2)^{2}(n-1) !}\|V\|_{2}^{2 n-2},
$$

where $s_{H}=\min \left\{\lambda_{k}-\lambda_{j}: 1 \leq j<k \leq m\right\}$.

On the strength of Theorem 2.10 we have the following reformulation of Question 3.1 and its relaxed alternative.

Question 3.9 Let $H, V \in \mathcal{M}_{d}(\mathbb{R})$, let $n \geq 2$.

(a) Find the largest constant $c_{n, d} \geq 0$ satisfying the bound

$$
\left\|\eta_{n}\right\|_{1} \geq c_{n, d}\|V\|_{2}^{n}
$$

(b) Is there an upper bound for $\|V\|_{2}$ in terms of $\eta_{1}, \ldots, \eta_{n}$ ?

Question 3.9 might be more approachable than Question 3.1 because explicit formulas for $\eta_{n}$ are known (see Theorem 2.11), though they are very complex. In [16, Theorems 3.4 and 3.6] upper bounds for $\|V\|_{2}$ were derived in terms of sparsity of the diameter of $\sigma(H)$ and the $L^{1}$ - and $L^{\infty}$-norms of the spectral shift functions.

\subsection{Reconstruction of a Perturbation}

The result of Theorem 3.4 can be viewed as a rudimentary inverse problem on reconstruction of a perturbation from values of different order Taylor remainders. It would be interesting to establish whether a nonzero perturbation can be reconstructed from the spectral shift functions.

While $\eta_{n}=0$ for $n>1$ determines $V$ uniquely, nonzero $\eta_{n}$ are invariant with respect to similarity transformations of $V$ by unitaries commuting with $H$. The following useful observation is due to [17, Proposition 4.7].

Proposition 3.10 Let $H, V \in \mathcal{M}_{d}(\mathbb{R})$. If $U \in \mathcal{M}_{d}$ is unitary and satisfies

$$
U H=H U,
$$


then

$$
\eta_{n, H, U^{*} V U}=\eta_{n, H, V} \text { a.e. }
$$

In view of Proposition 3.10, the nonzero uniqueness could possibly hold only up to unitary transformations satisfying (3.7).

Question 3.11 Let $H, V \in \mathcal{M}_{d}(\mathbb{R})$. Is $V$ determined uniquely up to an $H$-commuting unitary equivalence (see (3.7)) by a finite or infinite number of not necessarily zero functions $\eta_{k, H, V}$ ?

Remark 3.12 If a certain collection $\left\{\eta_{k, H, V}\right\}_{k}$ does not determine $V$ uniquely up to an $H$-commuting unitary equivalence in the case of most general $H, V \in \mathcal{M}_{d}(\mathbb{R})$, it might still determine $V$ uniquely under the additional assumption on the structure of $H$ and $H+V$. That is, Question 3.11 can also be addressed in the case of structured matrices $H$ and $H+V$, for instance, adjacency matrices of graphs.

\section{Positivity Preserving Property of Schur Multipliers}

In this section we collect partial results and open questions on characterization of the positive semi-definiteness of Schur multipliers in terms of analogous properties of their integrands.

\subsection{Positivity of Taylor Remainders}

The positivity preserving property of a Taylor remainder of a matrix function was first addressed in the influential paper [1]. In particular, the following characterization of monotone matrix functions in terms of the respective scalar functions is due to [1].

We assume below that $-\infty \leq a<b \leq \infty$.

Theorem 4.1 Let $f \in C^{1}(a, b)$ be real-valued. Then, $f(H+V)-f(H) \geq 0$ for all $H \in \mathcal{M}_{d}(a, b)$ and $V \in \mathcal{M}_{d}[0, \infty)$ such that $H+V \in \mathcal{M}_{d}(a, b)$ if and only if $\left(f^{[1]}\left(x_{i}, x_{j}\right)\right)_{i, j=1}^{d} \geq 0$ for all $\left\{x_{i}\right\}_{i=1}^{d} \subset(a, b)$.

The characterization of matrix monotone functions was followed by the characterization of matrix convex function in [18].

Theorem 4.2 Let $f \in C^{2}(a, b)$ be real-valued. Then, $f(H+V)-f(H)-\frac{\mathrm{d}}{\mathrm{d} t} f(H+$ $t V)\left.\right|_{t=0} \geq 0$ for all $H \in \mathcal{M}_{d}(a, b)$ and $V \in \mathcal{M}_{d}[0, \infty)$ such that $H+V \in \mathcal{M}_{d}(a, b)$ if and only if $\left(f^{[2]}\left(x_{i}, x_{j}, x_{1}\right)\right)_{i, j=1}^{d} \geq 0$ for all $\left\{x_{i}\right\}_{i=1}^{d} \subset(a, b)$.

An attempt to obtain a similar characterization of functions whose higher order matrix Taylor remainders preserve the sign of a perturbation was made in [19]. The following result is a consequence of [19, Theorem 2.1 and Proposition 2.6]. 
Theorem 4.3 Let $n \in \mathbb{N}$ and let $f \in C^{n}(a, b)$ be real-valued. Assume that

$$
f(H+V)-f(H)-\left.\sum_{k=0}^{n-1} \frac{1}{k !} \frac{\mathrm{d}^{k}}{\mathrm{~d} t^{k}} f(H+t V)\right|_{t=0} \geq 0
$$

for all $H \in \mathcal{M}_{d}(a, b)$ and $V \in \mathcal{M}_{d}[0, \infty)$ such that $H+V \in \mathcal{M}_{d}(a, b)$. Then,

$$
\left(f^{[n]}(x_{i}, x_{j}, \underbrace{x_{1}, \ldots, x_{1}}_{n-1})\right)_{i, j=1}^{d} \geq 0
$$

for all $\left\{x_{i}\right\}_{i=1}^{d} \subset(a, b)$.

Question 4.4 Let $n \in \mathbb{N}, n \geq 3$, and let $f \in C^{n}(a, b)$. Does (4.2) imply (4.1)?

By the equivalence of (4.1) to an analogous property of the $n$th order derivative of a matrix function verified in [19, Proposition 2.6] and by the representation for the derivative (2.11), we obtain the following consequences of Theorems 4.1, 4.2, and 4.3.

Corollary 4.5 Let $n \in \mathbb{N}$ and let $f \in C^{n}(a, b)$ be real-valued. If

$$
T_{f^{[n]}}^{H, \ldots, H}(V, \ldots, V) \geq 0
$$

for all $H \in \mathcal{M}_{d}(a, b)$ and $V \in \mathcal{M}_{d}[0, \infty)$ such that $H+V \in \mathcal{M}_{d}(a, b)$, then (4.2) holds. If $n=1,2$, then (4.2) implies (4.3).

In addition to the algebraic characterization stated in Theorem 4.1, matrix monotone functions were also characterized analytically in [1]. Subsequently, functions whose matrix Taylor remainders satisfy the positivity preserving property were characterized analytically in [19, Theorem 3.3 and Corollary 3.4] as stated in the theorem below.

Theorem 4.6 Let $f \in C^{n}(a, b)$ be real-valued. Then, (4.3) holds for all $d \in \mathbb{N}$, for all $H \in \mathcal{M}_{d}(a, b)$ and $V \in \mathcal{M}_{d}[0, \infty)$ such that $H+V \in \mathcal{M}_{d}(a, b)$ if and only if

$$
f(x)=\sum_{k=0}^{n-2} \frac{f^{(k)}\left(x_{0}\right)}{k !}\left(x-x_{0}\right)^{k}+\left(x-x_{0}\right)^{n-1} g(x),
$$

where $g$ satisfies Theorem 4.1 for every $d \in \mathbb{N}$ (that is, $g$ is matrix monotone for every matrix dimension).

\subsection{Positivity of Schur multipliers}

Theorems 4.1 and 4.2 admit the following analogs for linear and bilinear Schur multiplier with general symbols.

Proposition 4.7 Let $n \in\{1,2\}$ and let $\mathfrak{m}(n)=\left\{m_{j_{0}, \ldots, j_{n}}\right\}_{j_{0}, \ldots, j_{2}=1}^{d}$. Then, the following assertions hold. 
(i) $\mathfrak{M}_{\mathfrak{m}(1)}(X) \geq 0$ for every $X \in \mathcal{M}_{d}[0, \infty)$ if and only if $\left(m_{i, k}\right)_{i, k=1}^{d} \in \mathcal{M}_{d}[0, \infty)$.

(ii) $\mathfrak{M}_{\mathfrak{m}(2)}(X, X) \geq 0$ for every $X \in \mathcal{M}_{d}(\mathbb{R})$ if and only of $\left(m_{i, j, k}\right)_{i, k=1}^{d} \in$ $\mathcal{M}_{d}[0, \infty)$ for every $j=1, \ldots, d$.

Proof (i) By the Schur product theorem (see, e.g., [20, Theorem 7.5.3]), $\mathfrak{M}_{\mathfrak{m}(1)}(X) \geq$ 0 for all $X,\left(m_{i, k}\right)_{i, k=1}^{d} \in \mathcal{M}_{d}[0, \infty)$. Conversely, applying the property $\mathfrak{M}_{\mathfrak{m}(1)}(X) \geq$ 0 to $X \in \mathcal{M}_{d}[0, \infty)$ with $x_{i j}=1, i, j=1, \ldots, d$, implies

$$
0 \leq\left\langle\mathfrak{M}_{\mathfrak{m}(1)}(X) \eta, \eta\right\rangle=\sum_{i, k=1}^{d} m_{i k} x_{i k} \bar{\eta}_{i} \eta_{k}=\left\langle\left(m_{i, k}\right)_{i, k=1}^{d} \eta, \eta\right\rangle, \quad \eta \in \mathbb{C}^{d}
$$

(ii) Assume that $\mathfrak{M}_{\mathfrak{m}(2)}(X, X) \geq 0$ for every $X \in \mathcal{M}_{d}(\mathbb{R})$. Let $\xi \in \mathbb{C}^{d}$ be such that $\xi_{i} \neq 0, i=1, \ldots, d$, and consider $X=\xi^{*} \xi$. Let $\eta \in \mathbb{C}^{d}$. Set $\tilde{\eta}_{i}=\eta_{i} / \xi_{i}$, $i=1, \ldots, d$. By direct calculations we obtain

$$
0 \leq\left\langle\mathfrak{M}_{\mathfrak{m}(2)}\left(\xi^{*} \xi, \xi^{*} \xi\right) \tilde{\eta}, \tilde{\eta}\right\rangle=\sum_{j=1}^{d} \sum_{i, k=1}^{d} m_{i, j, k} \bar{\eta}_{i} \eta_{k}\left|\xi_{j}\right|^{2}
$$

Applying the above formula to a sequence $\left\{\xi_{l}^{(m)}\right\}_{m=1}^{\infty}$ satisfying

$$
\lim _{m \rightarrow \infty} \xi_{l}^{(m)}= \begin{cases}1 & \text { if } l=j \\ 0 & \text { if } l \neq j\end{cases}
$$

implies

$$
\left\langle\left(m_{i, j, k}\right)_{i, k=1}^{d} \eta, \eta\right\rangle=\sum_{i, k=1}^{d} m_{i, j, k} \bar{\eta}_{i} \eta_{k} \geq 0, \quad j=1, \ldots, d, \quad \eta \in \mathbb{C}^{d}
$$

Suppose now that $\left(m_{i, j, k}\right)_{i, k=1}^{d} \in \mathcal{M}_{d}[0, \infty)$ for every $j=1, \ldots, d$. Then,

$$
\sum_{i, k=1}^{d} m_{i, j, k} \overline{\xi_{i} \eta_{i}} \xi_{k} \eta_{k} \geq 0
$$

for all $\xi, \eta \in \mathbb{C}^{d}$ and $j=1, \ldots, d$. Let $X \in \mathcal{M}_{d}(\mathbb{R})$ and fix $j \in\{1, \ldots, d\}$. Applying (4.4) to $\xi_{i}=x_{j i}$ yields

$$
\sum_{i, k=1}^{d} m_{i, j, k} x_{i j} x_{j k} \bar{\eta}_{i} \eta_{k} \geq 0
$$


Summing over $j=1, \ldots, d$ in (4.5) implies

$$
\left\langle\mathfrak{M}_{\mathfrak{m}(2)}(X, X) \eta, \eta\right\rangle=\sum_{j=1}^{d} \sum_{i, k=1}^{d} m_{i, j, k} x_{i j} x_{j k} \bar{\eta}_{i} \eta_{k} \geq 0, \quad \eta \in \mathbb{C}^{d}
$$

Question 4.8 (i) Let $n$ be odd. Characterize the property " $\mathfrak{M}_{\mathfrak{m}(n)}(X, \ldots, X) \geq 0$ for every $X \in \mathcal{M}_{d}[0, \infty)$ " in terms of properties of $\mathfrak{m}(n)$.

(ii) Let $n$ be even. Characterize the property " $\mathfrak{M}_{\mathfrak{m}(n)}(X, \ldots, X) \geq 0$ for every $X \in$ $\mathcal{M}_{d}(\mathbb{R})$ " in terms of properties of $\mathfrak{m}(n)$.

\section{Tracial positivity of Schur multipliers}

The positivity preserving and positivity improving properties of the trace of a matrix function Taylor remainder pertain to an open problem in mathematical physics on the analogous properties of the spectral shift functions.

\subsection{Positivity of Spectral Shift Functions}

Question 5.1 Let $H, V \in \mathcal{M}_{d}(\mathbb{R})$ and $n \in \mathbb{N}, n \geq 3$.

(i) Does $V \geq 0$ imply that $\eta_{n, H, V} \geq 0$ ?

(ii) Assume, in addition, that $n$ is even. Does the property $\eta_{n, H, V} \geq 0$ hold?

The answers to Question 5.1 with $n=1,2$ are known to be affirmative. In the infinite-dimensional case they are due to [21] and [13], respectively. Different proofs of the respective results in the finite-dimensional case can be found in [14, Theorem 6.1 and Lemma 5.6] and [22, Proposition 3.3]. As to the case $n \geq 3$, we only have partial results summarized below.

The following result is a particular case of [22, Theorem 1.4].

Theorem 5.2 Let $H, V \in \mathcal{M}_{d}(\mathbb{R})$, let $[a, b]$ be the convex hull of $\sigma(H)$, and let $n \in \mathbb{N}$. Then, the following assertions hold.

(i) If $n$ is odd and $V \geq 0$ (respectively, $V \leq 0$ ), then $\eta_{n}(t) \geq 0$ (respectively, $\left.\eta_{n}(t) \leq 0\right)$ for a.e. $t \in \mathbb{R} \backslash[a, b]$.

(ii) If $n$ is even, then $\eta_{n}(t) \geq 0$ for a.e. $t \in \mathbb{R} \backslash[a, b]$.

(iii) If $V \geq 0$, then $\eta_{n}(t)=0$ for a.e. $t<$ a. If $V \leq 0$, then $\eta_{n}(t)=0$ for a.e. $t>b$.

The result below is due to [22, Corollary 1.6].

Corollary 5.3 Let $H, V \in \mathcal{M}_{d}(\mathbb{R})$ and $n \in \mathbb{N}$. Assume that either $V$ is rank-one or $d=2$. Then, the following assertions hold.

(i) Let $n$ be odd. If $V \geq 0$ (respectively, $V \leq 0$ ), then $\eta_{n} \geq 0$ (respectively, $\eta_{n} \leq 0$ ).

(ii) Let $n$ be even. If $V \geq 0$ or $V \leq 0$, then $\eta_{n} \geq 0$. 
In view of Theorem 2.10, Question 5.1 admits the following equivalent reformulation.

Question 5.4 Let $H, V \in \mathcal{M}_{d}(\mathbb{R})$, let $[a, b]$ be the convex hull of $\sigma(H) \cup \sigma(H+V)$, and let $n \in \mathbb{N}, n \geq 3$. Let $S \subset C^{n}[a, b]$ be such that $\left\{f^{(n)}: f \in S\right\}$ is dense in $C[a, b]$.

(i) Does $V \geq 0$ imply $\operatorname{Tr}\left(R_{n, H, V}(f)\right) \geq 0$ for every $f \in S$ ?

(ii) Assume, in addition, that $n$ is even. Does the property $\operatorname{Tr}\left(R_{n, H, V}(f)\right) \geq 0$ hold for every $f \in S$ ?

\subsection{Tracial Positivity of Schur Multipliers with Nonnegative Symbols}

We consider the latter property since it is related to the positivity of spectral shift functions on the strength of the representation (2.12) (see Theorem 2.6).

Tracial positivity of the Schur multipliers (2.3) with entrywise positive symbols $\varphi$ and sign-definite perturbations was established in [22] under several additional assumptions stated below.

It follows from (2.4) that $\operatorname{Tr}\left(T_{\varphi}^{H, \ldots, H}(X, \ldots, X)\right) \geq 0$ when $\varphi \geq 0$ and $\omega_{n} \geq 0$, where the measure $\omega_{n}$ is defined in (2.18). The following sufficient conditions for $\operatorname{Tr}\left(T_{\varphi}^{H, \ldots, H}(X, \ldots, X)\right) \geq 0$ are inherited from the sufficient conditions for $\omega_{n} \geq 0$ obtained in [22, Propositions 3.5 and 3.6] in the case $n \geq 3$ and follow from a straightforward observation in the case $n=2$.

Proposition 5.5 Let $H \in \mathcal{M}_{d}(\mathbb{R})$ and $X \in \mathcal{M}_{d}$. Let $n \in \mathbb{N}$ and $\varphi: \mathbb{R}^{n+1} \rightarrow[0, \infty)$. Then, the following assertions hold.

(i) If $n=2$, then $\operatorname{Tr}\left(T_{\varphi}^{H, H, H}\left(X, X^{*}\right)\right) \geq 0$.

(ii) Assume that $\sigma(H)=\left\{\lambda_{1}<\lambda_{2}\right\}$.

(a) If $X \in \mathcal{M}_{d}[0, \infty) \cup \mathcal{M}_{d}(-\infty, 0]$, then

$$
\operatorname{Tr}\left(T_{\varphi}^{H, \ldots, H}(\underbrace{X, \ldots, X}_{n})\right) \geq 0
$$

for $n \in\{4,6\}$.

(b) Assume, in addition, that at least one of $\lambda_{1}, \lambda_{2}$ has multiplicity one. Then, (5.1) holds for every even $n$.

(iii) Assume that $\sigma(H)=\left\{\lambda_{1}<\lambda_{2}\right\}$.

(a) If $X \in \mathcal{M}_{d}[0, \infty)$, then (5.1) holds for $n \in\{3,5\}$.

(b) Assume, in addition, that at least one of $\lambda_{1}, \lambda_{2}$ has multiplicity one. Then, (5.1) holds for every odd $n$.

(iv) Assume that $X \in \mathcal{M}_{d}(\mathbb{R})$ is rank-one. If $n$ is odd, assume also that $X \in$ $\mathcal{M}_{d}[0, \infty)$. Then, (5.1) holds for every $n$.

The tracial positivity of Schur multipliers on symmetric tuples was studied in [23] without imposing restrictions on the spectra of $H$ and $X$. 
Notations 5.6 Let $n \in \mathbb{N}, n \geq 2$. Given the multidimensional matrix

$$
\mathfrak{m}(n)=\left\{m_{j_{1}, \ldots, j_{n+1}}\right\}_{j_{1}, \ldots, j_{n+1}=1}^{d},
$$

denote the matrices

$$
\begin{aligned}
& M_{\mathfrak{m}(2)}:=\left(m_{j_{1}, j_{2}, j_{1}}\right)_{j_{2}, j_{1}=1}^{d}, \\
& M_{\mathfrak{m}(3)}^{\left(j_{2}\right)}:=\left(m_{j_{1}, j_{2}, j_{3}, j_{1}}\right)_{j_{3}, j_{1}=1}^{d}, \\
& M_{\mathfrak{m}(4)}^{\left(j_{2}, j_{4}\right)}:=\left(m_{j_{1}, j_{2}, j_{3}, j_{4}, j_{1}}\right)_{j_{3}, j_{1}=1}^{d} \quad \text { if } n \geq 4,
\end{aligned}
$$

where $j_{2}, j_{4} \in\{1, \ldots, d\}$.

Let $\pi$ be a cyclic permutation of $n$ elements, denote by $\pi^{-1}$ its inverse, and by $\pi_{k}(1, \ldots, n)$ the $k$ th component of the tuple $\pi(1, \ldots, n)$. Given an ordered tuple of objects $\left(z_{1}, \ldots, z_{n}\right)$, denote

$$
\pi\left(z_{1}, \ldots, z_{n}\right):=\left(z_{\pi_{1}(1, \ldots, n)}, \ldots, z_{\pi_{n}(1, \ldots, n)}\right) .
$$

Let $\pi(\mathfrak{m}(n))$ be the multidimensional matrix with the entries

$$
\pi(\mathfrak{m}(n))_{\pi\left(j_{1}, \ldots, j_{n}\right), j_{\pi_{1}(1, \ldots, n)}}:=m_{j_{1}, \ldots, j_{n}, j_{1}} .
$$

The following result is due to [23, Theorem 4.6].

Theorem 5.7 Let $n \in \mathbb{N}, n \geq 2$, and let $H \in \mathcal{M}_{d}(\mathbb{R})$. Assume Notations 2.1 and 5.6. Then, the following assertions hold.

(i) If $\varphi\left(\lambda_{i}, \lambda_{i}\right) \geq 0$ for all $i=1, \ldots, d$ and $X \in \mathcal{M}_{d}[0, \infty)$, then $\operatorname{Tr}\left(T_{\varphi}^{H, H}(X)\right) \geq$ 0 .

(a) Let $M_{\mathfrak{m}(2)} \in \mathcal{M}_{d}(\mathbb{R})$. Then, $\operatorname{Tr}\left(T_{\varphi}^{H, H, H}(X, Y)\right) \geq 0$ for all $X, Y \in$ $\mathcal{M}_{d}[0, \infty)$ if and only if $M_{\mathfrak{m}(2)} \in \mathcal{M}_{d}[0, \infty)$.

(b) If all entries of $M_{\mathfrak{m}(2)}$ are nonnegative, then $\operatorname{Tr}\left(T_{\varphi}^{H, H, H}\left(X, X^{*}\right)\right) \geq 0$.

(ii) Let $M_{\pi(\mathfrak{m}(3))}^{(j)} \in \mathcal{M}_{d}(\mathbb{R}), j=1, \ldots, d$. Then, $\operatorname{Tr}\left(T_{\varphi}^{H, \ldots, H}\left(\pi^{-1}\left(X^{*}, X, Y\right)\right)\right) \geq 0$ for all $X \in \mathcal{M}_{d}, Y \in \mathcal{M}_{d}[0, \infty)$ if and only if $M_{\pi(\mathfrak{m}(3))}^{(j)} \in \mathcal{M}_{d}[0, \infty)$ for all $j=1, \ldots, d$.

(a) Let $M_{\pi(\mathfrak{m}(4))}^{(j, l)} \in \mathcal{M}_{d}(\mathbb{R}), j, l=1, \ldots, d$. Then,

$\operatorname{Tr}\left(T_{\varphi}^{H, \ldots, H}\left(\pi^{-1}\left(X^{*}, X, Y^{*}, Y\right)\right)\right) \geq 0$ for all $X, Y \in \mathcal{M}_{d}$ if and only if $M_{\pi(\mathfrak{m}(4))}^{(j, l)} \in \mathcal{M}_{d}[0, \infty)$ for all $j, l=1, \ldots, d$.

(b) If all entries of $M_{\pi(\mathfrak{m}(4))}^{(j, j)}$ are nonnegative for all $j=1, \ldots, d$ and $\pi(\mathfrak{m}(4))_{i, j, k, l, i}=0$ for $j \neq l$, then $\operatorname{Tr}\left(T_{\varphi}^{H, \ldots, H}\left(\pi^{-1}\left(X, Y, Y^{*}, X^{*}\right)\right)\right) \geq 0$.

The tracial positivity of $n$-linear Schur multipliers on partially symmetric tuples with mildly sparse symbols $\mathfrak{m}(n), n \geq 5$, was characterized in [23, Theorem 4.6]. The interested reader can consult the given reference for details. 


\section{Tracial Bounds for Schur Multipliers}

In this section we discuss sharpening both lower and upper bounds for traces of Schur multipliers.

The bound (2.6) stated in Corollary 2.4 cannot generally be improved even in the case of commuting matrices. Indeed, let $X_{1}=\cdots=X_{n}=X=\operatorname{diag}(1,0, \ldots, 0)$, let $H=\operatorname{diag}(\lambda, 0, \ldots, 0)$, and let $\varphi_{\text {diag }}(\lambda)=\varphi(\lambda, \ldots, \lambda)$. Then,

$$
\operatorname{Tr}\left(T_{\varphi}^{H, \ldots, H}(X, \ldots, X)\right)=\operatorname{Tr}\left(\varphi_{\text {diag }}(H) X^{n}\right)=\varphi(\lambda, \ldots, \lambda)\|X\|_{2}^{n}
$$

Thus, the equality can be attained in (2.6) for a suitable choice of $\varphi$ and $\lambda$. Nonetheless, (2.6) has been sharpened in a number of cases discussed below. The following twosided bounds for traces of multilinear Schur multipliers are due to [23, Theorem 4.4].

Theorem 6.1 Let $n \in \mathbb{N}, n \geq 2$, and let $H \in \mathcal{M}_{d}(\mathbb{R})$. Assume Notations 2.1 and 5.6. Let $\left\{\lambda_{i}\right\}_{i=1}^{d}$ be the complete list of eigenvalues of $H$ and let $U$ be a unitary operator such that $U H U^{*}=\operatorname{diag}\left(\lambda_{1}, \ldots, \lambda_{d}\right)$. Then, the following assertions hold.

(i) If $\varphi\left(\lambda_{i}, \lambda_{i}\right) \geq 0$ for all $i=1, \ldots, d$ and $X \in \mathcal{M}_{d}[0, \infty)$, then

$$
\min _{1 \leq i \leq d} \varphi\left(\lambda_{i}, \lambda_{i}\right) \cdot \operatorname{Tr}(X) \leq \operatorname{Tr}\left(T_{\varphi}^{H, H}(X)\right) \leq \max _{1 \leq i \leq d} \varphi\left(\lambda_{i}, \lambda_{i}\right) \cdot \operatorname{Tr}(X) .
$$

(ii) If $M_{\mathfrak{m}(2)} \in \mathcal{M}_{d}(\mathbb{R})$ and $X, Y \in \mathcal{M}_{d}[0, \infty)$, then

$$
\begin{aligned}
& \lambda_{\min }\left(M_{\mathfrak{m}(2)}\right) \cdot \operatorname{Tr}\left(\left(U X U^{*}\right) \circ\left(\bar{U} Y^{T} U^{T}\right)\right) \\
& \leq \operatorname{Tr}\left(T_{\varphi}^{H, \ldots, H}(X, Y)\right) \\
& \leq \lambda_{\max }\left(M_{\mathfrak{m}(2)}\right) \cdot \operatorname{Tr}\left(\left(U X U^{*}\right) \circ\left(\bar{U} Y^{T} U^{T}\right)\right) .
\end{aligned}
$$

(iii) If $M_{\pi(\mathfrak{m}(3))}^{(j)} \in \mathcal{M}_{d}(\mathbb{R})$ for all $j=1, \ldots, d, X \in \mathcal{M}_{d}$, and $Y \in \mathcal{M}_{d}[0, \infty)$, then

$$
\begin{aligned}
& \min _{1 \leq j \leq d} \lambda_{\min }\left(M_{\pi(\mathfrak{m}(3))}^{(j)}\right) \cdot \operatorname{Tr}\left(\left(U|X|^{2} U^{*}\right) \circ\left(U Y U^{*}\right)\right) \\
& \leq \operatorname{Tr}\left(T_{\varphi}^{H, \ldots, H}\left(\pi^{-1}\left(X^{*}, X, Y\right)\right)\right) \\
& \leq \max _{1 \leq j \leq d} \lambda_{\max }\left(M_{\pi(\mathfrak{m}(3))}^{(j)}\right) \cdot \operatorname{Tr}\left(\left(U|X|^{2} U^{*}\right) \circ\left(U Y U^{*}\right)\right) .
\end{aligned}
$$

(iv) If $M_{\pi(\mathfrak{m}(4))}^{(j, l)} \in \mathcal{M}_{d}(\mathbb{R})$ for all $j, l=1, \ldots, d$ and $X, Y \in \mathcal{M}_{d}$, then

$$
\begin{aligned}
& \min _{\{(j, l): 1 \leq j, l \leq d\}} \lambda_{\min }\left(M_{\pi(\mathfrak{m}(4))}^{(j, l)}\right) \cdot \operatorname{Tr}\left(\left(U|X|^{2} U^{*}\right) \circ\left(U|Y|^{2} U^{*}\right)\right) \\
& \leq \operatorname{Tr}\left(T_{\varphi}^{H, \ldots, H}\left(\pi^{-1}\left(X^{*}, X, Y^{*}, Y\right)\right)\right) \\
& \leq \max _{\{(j, l): 1 \leq j, l \leq d\}} \lambda_{\max }\left(M_{\pi(\mathfrak{m}(4))}^{(j, l)}\right) \cdot \operatorname{Tr}\left(\left(U|X|^{2} U^{*}\right) \circ\left(U|Y|^{2} U^{*}\right)\right) .
\end{aligned}
$$


Analogous results for mildly sparse symbols $\mathfrak{m}(n), n \geq 5$, were obtained in [23, Theorem 4.4]. Below we compare the results of Theorem 6.1 and Corollary 2.4 following [23, Remark 4.5].

In certain cases when the smallest and largest eigenvalues of $M_{\mathfrak{m}(2)}, M_{\pi(\mathfrak{m}(3))}^{(j)}$, $M_{\pi(\mathfrak{m}(4))}^{(j, l)}$ are not symmetric about the origin, the estimates obtained in Theorem 6.1 give a more accurate dependence on the symbol $\pi(\mathfrak{m}(n))$ than (2.6). That occurs when, for instance, one of the following properties is satisfied:

(a) $\lambda_{\min }(M)>-\|M\|_{\ell_{\infty}\left(\mathbb{C}^{d^{2}}\right)} \geq-\|M\|=-\lambda_{\max }(M)$ for the first bound,

(b) $\lambda_{\min }(M) \geq 0$ for the first bound,

(c) $\lambda_{\max }(M)<\|M\|_{\ell_{\infty}\left(\mathbb{C}^{d^{2}}\right)} \leq\|M\|=-\lambda_{\min }(M)$ for the second bound,

(d) $\lambda_{\max }(M) \leq 0$ for the second bound,

where $M \in\left\{M_{\mathfrak{m}(2)}, M_{\pi(\mathfrak{m}(3))}^{(j)}, M_{\pi(\mathfrak{m}(4))}^{(j, l)}\right\}$. We note that the second inequality in (a) and (c) is based on the property $\|B\|_{\ell_{\infty}\left(\mathbb{C}^{d^{2}}\right)} \leq\|B\|$ satisfied by every $B \in \mathcal{M}_{d}$ (see, e.g., [20, Sect. 5.6, Problem 23]).

The dependence on the input matrices $X, Y$ in Theorem 6.1(ii)-(iv) is more accurate than the one in (2.6) with the same inputs when, for instance, either $\lambda_{\min }(M) \leq 0$ (for the first bound) or $\lambda_{\max }(M) \geq 0$ (for the second bound) because, as noted in [23, Remark 4.5],

$$
\begin{aligned}
& \operatorname{Tr}\left(\left(U X U^{*}\right) \circ\left(\bar{U} Y^{T} U^{T}\right)\right) \leq\|X\|_{2}\|Y\|_{2}, \\
& \operatorname{Tr}\left(\left(U|X|^{2} U^{*}\right) \circ\left(U Y U^{*}\right)\right) \leq\|X\|_{4}^{2}\|Y\|_{2} \leq\|X\|_{2}^{2}\|Y\|_{2}, \\
& \operatorname{Tr}\left(\left(U|X|^{2} U^{*}\right) \circ\left(U|Y|^{2} U^{*}\right)\right) \leq\|X\|_{4}^{2}\|Y\|_{4}^{2} \leq\|X\|_{2}^{2}\|Y\|_{2}^{2} .
\end{aligned}
$$

Based on the observations made above we conclude that the estimates obtained in Theorem 6.1(ii)-(iv) improve the estimate (2.6) in, for instance, each of the following cases:

(1) the first bound when (a) holds and $\lambda_{\min }(M) \leq 0$,

(2) the first bound when $\lambda_{\min }(M) \geq 0$,

(3) the second bound when (c) holds and $\lambda_{\max }(M) \geq 0$,

(4) the second bound when $\lambda_{\max }(M) \leq 0$.

Question 6.2 Sharpen the results of [23, Theorem 4.4] and, if possible, the results of Theorem 6.1.

\section{Declarations}

Conflict of interest The author has no relevant financial or non-financial interests to disclose.

\section{References}

1. Löwner, K.: Über monotone Matrixfunktionen. Math. Z. 38(1), 177-216 (1934) (in German) 
2. Daletskii, Yu.L., Krein, S.G.: Integration and differentiation of functions of Hermitian operators and application to the theory of perturbations. Trudy Sem. Functsion. Anal., Voronezh. Gos. Univ. 1, 81-105 (1956) (in Russian)

3. Skripka, A., Tomskova, A.: Multilinear Operator Integrals: Theory and Applications. Lecture Notes in Mathematics, vol. 2250. Springer, Cham (2019)

4. Skripka, A.: Untangling noncommutativity with operator integrals. Not. Am. Math. Soc. 67(1), 45-55 (2020)

5. Birman, M.S., Solomyak, M.Z.: Double operator integrals in a Hilbert space. Integral Equ. Oper. Theory 47(2), 131-168 (2003)

6. Juschenko, K., Todorov, I.G., Turowska, L.: Multidimensional operator multipliers. Trans. Am. Math. Soc. 361, 4683-4720 (2009)

7. Peller, V.V.: Multiple operator integrals in perturbation theory. Bull. Math. Sci. 6, 15-88 (2016)

8. Effros, E.G., Ruan, Z.-J.: Multivariable multipliers for groups and their operator algebras. In: Proceedings of Symposium on Pure Mathematics, vol. 51, Part 1, pp. 197-218. American Mathematical Society, Providence (1990)

9. Potapov, D., Skripka, A., Sukochev, F.: Spectral shift function of higher order. Invent. Math. 193(3), 501-538 (2013)

10. Potapov, D., Skripka, A., Sukochev, F., Tomskova, A.: Multilinear Schur multipliers and applications to operator Taylor remainders. Adv. Math. 320, 1063-1098 (2017)

11. Lifshits, I.M.: On a problem of the theory of perturbations connected with quantum statistics. Uspehi Matem. Nauk (N.S.) 7(1(47)), 171-180 (1952) (in Russian)

12. Krein, M.G.: On a trace formula in perturbation theory. Matem. Sbornik 33, 597-626 (1953) (Russian)

13. Koplienko, L.S.: Trace formula for perturbations of nonnuclear type, Sibirsk. Mat. Zh. 25, 62-71 (1984) (in Russian). Translation: Siberian Math. J. 25, 735-743 (1984)

14. Skripka, A.: Trace inequalities and spectral shift. Oper. Matrices 3(2), 241-260 (2009)

15. Dykema, K., Skripka, A.: Higher order spectral shift. J. Funct. Anal. 257, 1092-1132 (2009)

16. Skripka, A., Zinchenko, M.: Stability and uniqueness properties of Taylor approximations of matrix functions. Linear Algebra Appl. 582, 218-236 (2019)

17. Skripka, A., Zinchenko, M.: On uniqueness of higher order spectral shift functions. Stud. Math. 251(2), 207-218 (2020)

18. Kraus, F.: Über konvexe Matrixfunktionen. Math. Z. 41(1), 18-42 (1936) (in German)

19. Franz, U., Hiai, F., Ricard, É.: Higher order extension of Löwner's theory: operator $k$-tone functions. Trans. Am. Math. Soc. 366(6), 3043-3074 (2014)

20. Horn, R.A., Johnson, C.R.: Matrix Analysis. Cambridge University Press, Cambridge (1985)

21. Birman, M.S., Solomyak, M.Z.: Remarks on the spectral shift function. J. Soviet Math. 3, 408-419 (1975)

22. Skripka, A.: On positivity of spectral shift functions. Linear Algebra Appl. 523, 118-130 (2017)

23. Skripka, A.: Tracial bounds for multilinear Schur multipliers. Linear Algebra Appl. 590, 62-84 (2020)

Publisher's Note Springer Nature remains neutral with regard to jurisdictional claims in published maps and institutional affiliations. 